\title{
A new action of the Kudo-Araki-May algebra on the dual of the symmetric algebras, with applications to the hit problem
}

\author{
DAVID PENGELLEY \\ FRANK WILLIAMS
}

\begin{abstract}
The hit problem for a cohomology module over the Steenrod algebra $\mathcal{A}$ asks for a minimal set of $\mathcal{A}$-generators for the module. In this paper we consider the symmetric algebras over the field $\mathbb{F}_{p}$, for $p$ an arbitrary prime, and treat the equivalent problem of determining the set of $\mathcal{A}^{*}$-primitive elements in their duals. We produce a method for generating new primitives from known ones via a new action of the Kudo-ArakiMay algebra $\mathcal{K}$, and consider the $\mathcal{K}$-module structure of the primitives, which form a sub $\mathcal{K}$-algebra of the dual of the infinite symmetric algebra. Our examples show that the $\mathcal{K}$-action on the primitives is not free. Our new action encompasses, on the finite symmetric algebras, the operators introduced by Kameko for studying the hit problem.
\end{abstract}

16T05, 16T10, 16W22, 55R45, 55S10, 55S12; 16W50, 55R40, 57T05, 57T10, 57T25

\section{Introduction and Theorem}

The hit problem for a cohomology module over the Steenrod algebra $\mathcal{A}$, for an arbitrary prime $p$, asks for a minimal set of $\mathcal{A}$-generators for the module, that is, a basis for its quotient by those elements hit under the left action of the positive dimensional elements of $\mathcal{A}$. For $p=2$ the hit problem for symmetric algebras has been studied by Janfada and Wood $[9 ; 10]$ (see below); and quotients had earlier been studied by Peterson [18]. The infinite symmetric algebra over a prime $p$ can be regarded as the cohomology $H^{*}\left(B U ; \mathbb{F}_{p}\right)$ (or $H^{*}\left(B O ; \mathbb{F}_{2}\right)$ if $p=2$ ). We shall adopt the point of view of Alghamdi, Crabb, and Hubbuck [1] and consider the equivalent problem of determining the set $\mathcal{P}$ of $\overline{\mathcal{A}}$-annihilated elements for the corresponding $\mathcal{A}$-action on the dual $M=H_{*}\left(B U ; \mathbb{F}_{p}\right)$ (or $H_{*}\left(B O ; \mathbb{F}_{2}\right)$ when $p=2$ ), that is, the kernel of the downward right action on $M$ of the positive dimensional elements of $\mathcal{A}$. These are also the primitive elements for the $\mathcal{A}^{*}$-coaction on $M$, and so we will henceforth call them primitives, as in literature such as Boardman [2], Bruner, Hà and Hung [3], Hung [8], and Powell [19]. The $H$-space multiplication on $B U$ (or $B O$ ) makes $M$ 
into a Hopf algebra, and the downward $\mathcal{A}$-action satisfies the Cartan formula, so the primitive elements $\mathcal{P}$ form a subalgebra of $M$.

We shall now state our main theorem, proved in Section 5, which provides a method of producing primitive elements for all primes, often indecomposable in the algebra $\mathcal{P}$, by defining an action of the Kudo-Araki-May bialgebra $\mathcal{K}[15 ; 16]$ on $M$. We emphasize for contrast that while the infinite loop space multiplication on $B U$ (or $B O$ ) makes $M$ into a Hopf algebra over the Dyer-Lashof algebra $\mathcal{R}$, or equivalently over the Kudo-Araki-May bialgebra $\mathcal{K}$, that action is very different from the one we define here (see Section 5 for the difference between the two $\mathcal{K}$-actions). We do not know a geometric interpretation for the new $\mathcal{K}$-action defined here.

Recall that $M$ is a polynomial algebra with generators $a_{n}(n \geq 1)$ dual to the powers $c_{1}^{n}$ (or $w_{1}^{n}$ if $p=2$ ) of the elementary symmetric function of degree one. (The reader should assume henceforth that the occurrence in any expressions of $a_{n}$ with $n \leq 0$ is interpreted as zero or omitted. In particular, note there is no nonzero element $a_{0}$.)

Theorem The formulas

$$
d_{0}(1)=1, \quad d_{i}(1)=0 \text { for } i>0,
$$

and for $n \geq 1$ and $i \geq 0$

$$
d_{i}\left(a_{n}\right)= \begin{cases}a_{n}^{p} & \text { if } i=0 \\ -a_{p n+(p-1)} & \text { if } i=1 \\ 0 & \text { if } i>1\end{cases}
$$

iterated and extended on products in $M$ by the Cartan formula using

$$
\Delta d_{m}=\sum_{i+j=m} d_{i} \otimes d_{j}
$$

respect the $\mathcal{K}$ Adem relations, and thus produce a graded action by the bialgebra $\mathcal{K}$ (equivalently $\mathcal{R}$ ) on the algebra $M$, where the $d_{i}$ are the standard generators of $\mathcal{K}$. The algebra $M$ thus becomes a $\mathcal{K}$-algebra, that is, an algebra over the bialgebra $\mathcal{K}$.

The action of $\mathcal{K}$ commutes up to Verschiebung with the right action of the Steenrod algebra, that is,

$$
\left(d_{i} x\right) * P^{k}=d_{i}\left(x * P^{k / p}\right) .
$$

Consequently, the subalgebra $\mathcal{P}$ of $M$ consisting of primitive elements is closed under the $\mathcal{K}$-action, hence becomes a sub-K $\mathcal{K}$-algebra. 
Our primary motivation for and application of this theorem is to generate new elements of $\mathcal{P}$ from known ones by applying elements of $\mathcal{K}$, and this we shall explore below in examples that use this new action to obtain and analyze families of primitive elements, especially at odd primes. It is nontrivial that the formulas above respect the Adem relations that determine $\mathcal{K}$, thus producing an action of $\mathcal{K}$ on $M$, rather than just of the free bialgebra on the elements $d_{i}$, and this will be exploited in our examples.

Singer [21] developed a method for finding primitives at the prime 2, by defining a 2 -primary "bigraded Steenrod algebra" with an action on $M$. This action is equivalent to the specialization of our action of $\mathcal{K}$ to the prime 2 , in a sense we shall explain at the end of the paper. And the examples of primitives we will produce for $p=2$ coincide with Singer's.

We shall also discuss how our action incorporates on the duals of the finite symmetric algebras the operators introduced by Kameko [11], Crossley [6], and Janfada and Wood $[9 ; 10]$ (see also Hà [7]).

\section{Background}

It is easy to solve the hit problem for the cohomology of a single projective space. Most work on the hit problem has been done on the cohomology of products of projective spaces (the canonical polynomial algebras over $\mathcal{A}$ on one-dimensional generators), where the problem is quite difficult. Initial results for such products were obtained by Peterson [17], and he made a conjecture [18] for $p=2$ about exactly which degrees contain $\mathcal{A}$-generators (albeit not their rank), which was proven by Wood [22]. A good reference list of the literature on attempts to solve the problem for projective spaces is given by Nam [13]. As we noted above, for $p=2$ the hit problem for symmetric algebras has been studied by Janfada and Wood $[9 ; 10]$.

Since the polynomial generators $a_{n}(n \geq 1)$ are the images of the positive degree elements of the homology of the projective space $\mathbb{C} P(\infty)=B U(1) \subset B U$ (or $\mathbb{R} P(\infty)=$ $B O(1) \subset B O$ ), by the Cartan formula the downward $\mathcal{A}$-action preserves the length of monomials in the generators $a_{n}$. So if we bigrade $M$ by length $l$ and topological degree $n$, we have

$$
P^{i}: M_{l, n} \rightarrow M_{l, n-(p-1) i}
$$

for the right action of the Steenrod powers from $\mathcal{A}$ (we use complex topological grading for $p$ odd, that is, $\left.a_{n} \in M_{1, n}=H_{2 n}\left(B U(1) ; \mathbb{F}_{p}\right)\right)$. Thus $M$ splits by length as an $\mathcal{A}$-module. Clearly too, $\mathcal{P}$ is a bigraded subalgebra of $M$.

We now review a few basic properties $[15 ; 16]$ of the Kudo-Araki-May algebra $\mathcal{K}$, which will be one of our main tools in this paper. The bigraded bialgebra $\mathcal{K}$ over a 
prime $p$ is generated by elements $d_{i} \in \mathcal{K}_{1,(p-1) i}, i=0,1, \ldots$ (if $p$ is odd we here too use the complex grading in the second coordinate) subject to their own set of (Adem) relations

$$
d_{i} d_{j}=\sum_{k}\left(\begin{array}{c}
(p-1)(k-j)-1 \\
p k-i-(p-1) j
\end{array}\right) d_{i+p j-p k} d_{k}, \quad(i>j) .
$$

The degree of elements in $\mathcal{K}$ satisfies the condition that multiplication is a map $\mathcal{K}_{l_{1}, r} \otimes \mathcal{K}_{l_{2}, n} \rightarrow \mathcal{K}_{l_{1}+l_{2}, p^{l_{1} n+r}}$. So the first index is the length of a monomial, and multiplication is skew-additive on the second index. N.B: The identity element is $1 \in \mathcal{K}_{0,0}$, quite distinct from $d_{0} \in \mathcal{K}_{1,0}$. A monomial $d_{I}=d_{i_{1}} \ldots d_{i_{l}}$ is admissible provided the multi-index $I=\left(i_{1}, \ldots, i_{l}\right)$ is nondecreasing, and we recall that the admissibles form a basis for $\mathcal{K}$.

A graded homology $\mathcal{K}$-module $N$ is one that satisfies the requirement $\mathcal{K}_{l, r} \otimes N_{n} \rightarrow$ $N_{p^{l} n+r}$. A graded homology $\mathcal{K}$-module structure on $N$ (complex graded if $p$ is odd) corresponds to an action of the Dyer-Lashof algebra $\mathcal{R}$ by iterating the conversion formula $Q^{i}\left(x_{n}\right)=(-1)^{i-n} d_{i-n}\left(x_{n}\right)$ on an element $x_{n} \in N_{n}$. Note that this is not merely a reindexing between $\mathcal{K}$ and $\mathcal{R}$, since the degree of the underlying homology element changes repeatedly during a composition. The algebra structures of $\mathcal{K}$ and $\mathcal{R}$ are very different.

We also recall that the algebra $\mathcal{K}$ has a compatible coproduct determined by

$$
\Delta d_{m}=\sum_{i+j=m} d_{i} \otimes d_{j}
$$

and that, just as the Dyer-Lashof action on the homology of an infinite loop space satisfies a Cartan formula on homology products using a coproduct in $\mathcal{R}$, so does the corresponding action of $\mathcal{K}$ via its own coproduct. We call such a combined structure of a graded homology $\mathcal{K}$-module with a product and a compatible Cartan formula a homology $\mathcal{K}$-algebra. Clearly the $\mathcal{K}$-action defined in our main theorem makes $M$ with its topological degree into a homology $\mathcal{K}$-algebra.

\section{Finite symmetric algebras}

From the definition, we see that $d_{i}: M_{l, n} \rightarrow M_{l+(p-1)(l-i), p n+(p-1) i}$. Examining the relations between $i$ and $l$ in this formula yields the following proposition.

Proposition 3.1 Applying $d_{l}$ to a monomial in $M$ of length $l$ in the polynomial generators $a_{k}$ preserves the length of the monomial, making the length $l$ subspaces of both $M$ and $\mathcal{P}$ into left $\mathbb{F}_{p}\left[d_{l}\right]$ modules as well as right $\mathcal{A}$-modules. In fact they 
are clearly each free modules over $\mathbb{F}_{p}\left[d_{l}\right]$, since $d_{l}$ is a monic endomorphism on the subspace of length $l$, and raises degree.

More generally, $d_{i}$ on a monomial of length $l$ is zero if $i>l$, of length $l$ if $i=l$, and of greater length if $i<l$.

We now see how this $\mathcal{K}$-module structure applies to finite symmetric algebras. The subspace of $M$ with basis the monomials in the $a_{n}$ of total length $\leq l$ corresponds to $H_{*}\left(B U(l) ; \mathbb{F}_{p}\right)$ (or $H_{*}\left(B O(l) ; \mathbb{F}_{2}\right)$ ), so the results in this paper also imply results on the primitives in these homology modules, and thus on the hit problem for their duals $H^{*}\left(B U(l) ; \mathbb{F}_{p}\right)$ (or $H^{*}\left(B O(l) ; \mathbb{F}_{2}\right)$ when $\left.p=2\right)$, the algebras of symmetric polynomials in finitely many variables; and similarly for the corresponding Thom spaces $M U(l)$ (or $M O(l)$ ), whose homology basis is the monomials of length exactly $l$. When $i<l$, the $\mathcal{K}$-action allows us to transfer information about primitives from each length degree to higher lengths. We will illustrate this in our examples of new families of primitive elements. The sparseness of the new $\mathcal{K}$-action formulas on generators (greatly simplified from the Dyer-Lashof action), combined with the Cartan formula on products, makes calculation relatively easy, as we shall see in the examples.

The fact that $\mathcal{P}$ is endowed with the structure of a module over $\mathcal{K}$ does more than simply produce new primitives from known ones. Specifically, the Adem relations in $\mathcal{K}$ will help reveal $\mathcal{K}$-connections between our examples of primitives in the module $\mathcal{P}$, and in the last section of the paper we will illustrate aspects of the $\mathcal{K}$-module structure, by showing that $\mathcal{P}$ has nontrivial $\mathcal{K}$-module relations.

At this point we note that our $\mathcal{K}$-action contains within it the duals on symmetric algebras of the operators $\psi_{k}$ introduced by Kameko [11] at the prime 2 for studying the hit problem for the cohomology of products of projective spaces. The operators $\psi_{k}$ are left inverses of his operators $\phi_{k}$ given by $\phi_{k}(f)=\sigma_{k} f^{2}$, which have been utilized by many researchers ( $\sigma_{k}$ is the $k$ th elementary symmetric function, that is, $c_{k}$ (or $w_{k}$ ) in our notation), and have been adapted for mod 2 symmetric algebras by Janfada and Wood [9; 10] (see also Crossley's map [6] analogous to $\psi_{2}^{*}$ for $H_{*}\left(\mathbb{C P}^{\infty} \times \mathbb{C P}^{\infty} ; \mathbb{F}_{p}\right)$ at odd primes).

We note that $\psi_{k}^{*}: M_{l, n} \rightarrow M_{k, 2 n+k}$ for $l \leq k$, and one can compute that

$$
\psi_{k}^{*}\left(a_{i_{1}} \ldots a_{i_{l}}\right)=a_{1}^{k-l} a_{2 i_{1}+1} \ldots a_{2 i_{l}+1}=a_{1}^{k-l} d_{l}\left(a_{i_{1}} \ldots a_{i_{l}}\right) .
$$

Thus on the length $k$ subspace $M_{k, *}$, we see that $\psi_{k}^{*}$ coincides exactly with $d_{k}$.

On the mod 2 homology of projective spaces, the dual Kameko operators $\psi_{k}^{*}$, each applied to the product of $k$ projective spaces, are combined together and called $\mathrm{Sq}^{0}$, 
and have been used to study the Singer transfer from the primitives to the cohomology of the Steenrod algebra (see Boardman [2], Bruner, Hà and Hung [3], Carlisle and Wood [4], Crabb and Hubbuck [5], Hà [7], Hung [8] and Singer [20; 21]). On the other hand, for the symmetric coinvariants, Singer has recently defined and extended the action of an analogous dual $\mathrm{Sq}^{0}$ of the Kameko operators to his action of a "bigraded Steenrod algebra" [21], and used it to find primitive elements. We explain at the end of the paper how Singer's action is equivalent to the $\mathcal{K}$-action above at the prime 2 .

Finally, let us consider the state of knowledge on the hit problem for symmetric algebras in $l$ variables. For $l=1$, the hit problem is easily solved for all primes (see below). For the prime 2 , the hit problem for symmetric algebras was solved for $l=2,3$ by Janfada and Wood [10]. We, on the other hand, shall use our $\mathcal{K}$-action to give examples for all primes $p$ of numerous families of primitives ranging over many lengths. For $p$ odd, these are entirely new. While some of these families specialize to zero at the prime 2 , those that remain nontrivial coincide with the elements given by Singer [21]. In a paper in preparation [14] we shall treat the hit problem of determining all the primitives in $H_{*}\left(B U(2) ; \mathbb{F}_{p}\right)$ at any prime, making use of Proposition 3.1. Additionally, Janfada and Wood [9] formulated and proved a Peterson conjecture for the finite symmetric algebras $H^{*}\left(B O(l) ; \mathbb{F}_{2}\right)$, which is formally identical, surprisingly, to the conjecture proven for products of projective spaces. At the end of this paper we will comment on an analogue at odd primes.

\section{Examples of primitives and of $\mathcal{K}$-module relations in $\mathcal{P}$}

In this section we shall illustrate some examples of primitives, showing how the $\mathcal{K}-$ action can be used to generate more primitives from existing ones, and also begin to see some nontrivial relations in the $\mathcal{K}$-module structure of $\mathcal{P}$, the algebra of primitives. Recall that $d_{0}$ always acts as the $p$ th power, so $p$ th powers of primitives always produce more primitives, and we will leave this largely unspoken in what follows. In fact we are particularly interested in finding new indecomposable primitives in $\mathcal{P}$.

Example 4.1 (Length one primitives) The primitives of length one are easily determined from the formula $a_{m} * P^{r}=\left(\begin{array}{c}m-r(p-1) \\ r\end{array}\right) a_{m-r(p-1)}$. A basis for $\mathcal{P}$ in length one is then

$$
\left\{a_{j p^{n-1}} \mid 1 \leq j \leq p-1, n \geq 0,(j, n) \neq(1,0)\right\} .
$$

And from this, since our $\mathcal{K}$-action formula says $d_{1}\left(a_{j p^{n}-1}\right)=-a_{j p^{n+1}-1}$, we obtain the set

$$
\left\{a_{k} \mid 1 \leq k \leq p-1\right\}
$$


as a basis for the free $\mathbb{F}_{p}\left[d_{1}\right]$-module structure that $\mathcal{P}$ has in length one. From Proposition 3.1 or the definition, $d_{i}$ for $i>1$ will be zero on the length one primitives, so this is the end of their story from the point of view of the $\mathcal{K}$-action.

Of course products of these length one primitives provide some higher length primitives in $\mathcal{P}$, but we shall now seek indecomposable primitives of higher lengths.

Example 4.2 Define

$$
\beta(k, l)=k a_{k} a_{l+p-1}-l a_{l} a_{k+p-1} \quad \text { for } \quad 1 \leq k<l \leq p-1 .
$$

(Note that this set is empty when $p=2$.)

Each of these length two elements is verified by inspection to be an indecomposable primitive in $\mathcal{P}$. Proposition 3.1 ensures that the length two primitives form a free $\mathbb{F}_{p}\left[d_{2}\right]$-module. We compute

$$
d_{2}^{n} \beta(k, l)=k a_{(k+1) p^{n}-1} a_{(l+p) p^{n-1}}-l a_{(l+1) p^{n}-1} a_{(k+p) p^{n-1}} .
$$

This example gives us many new primitive elements of monomial length two, which are dual to $\mathcal{A}$-indecomposables in $H^{*}\left(B U(2) ; \mathbb{F}_{p}\right)$. (We shall see in [14] that there are many more length two primitives.)

Of course the full $\mathcal{K}$-action on the $\beta(k, l)$ might yet produce more primitives, of higher length. Since $\mathcal{K}$ has as basis the admissible monomials, we need only consider action by admissibles. Combined with Proposition 3.1 and the fact that $d_{0}$ always acts as the $p$ th power, we see that to understand what the $\beta(k, l)$ can produce, it remains only to consider how $d_{1}$ acts on them, which will yield primitives of higher length. This we will do below.

\section{Example 4.3 Define}

$$
\alpha(k, l)=k a_{k}^{p} a_{p^{2}+l p-1}-l a_{k+p-1}^{p} a_{l p+p-1,}
$$

for $1 \leq k, l \leq p-1$. N.B: While for $\beta(k, l)$ we required $k<l$, here there is no such restriction. Note that $\alpha(k, l)$ has length $p+1$. A short calculation checks that all such $\alpha(k, l)$ are primitive. Clearly they are also indecomposable in $\mathcal{P}$. (Note that when $p=2, \alpha(1,1)=a_{1}^{2} a_{5}+a_{2}^{2} a_{3}$ is a length three indecomposable primitive in degree 7 , see Singer [21, page 559].)

Now let us explore further using the $\mathcal{K}$-action, to see what additional primitives may arise, and also to begin to see relations in the $\mathcal{K}$-module structure of $\mathcal{P}$. Since $\mathcal{K}$ has as basis the admissibles, we need only consider action by these and compare results to find $\mathcal{K}$-module relations. 
Consider first the action of $d_{1}$ on the set $\{\beta(k, l) \mid 1 \leq k<l \leq p-1\}$. The operation $d_{1}$ will raise their length from two to $p+1$, the same length as the $\alpha(k, l)$.

We observe that

$$
d_{1} \beta(k, l)=-\alpha(k, l)+\alpha(l, k),
$$

a linear combination of primitives already discovered above.

We examine the action of $\mathcal{K}$ on $\alpha(k, l)$. We see from the Cartan formula (refining Proposition 3.1) that $d_{i}(\alpha(k, l))=0$ unless $i=0,1, p$, or $p+1$. Further, for $s \geq 0$, one calculates easily that for $k<l$,

$$
d_{p} d_{p+1}^{s} \alpha(k, l)=-d_{0} d_{2}^{s+1} \beta(k, l),
$$

in length $2 p$, the $p$ th power of an element of length two from Example 4.2. Thus $d_{p} d_{p+1}^{s}(\alpha(k, l))$ does not produce new indecomposable elements of $\mathcal{P}$, but since it is admissibles in $\mathcal{K}$ that appear on both sides, this does display nontrivial $\mathcal{K}$-module relations in the structure of $\mathcal{P}$.

However, applying $d_{1}$ and $d_{p+1}$ to $\alpha(k, l)$ does yield new primitive elements. Iterating these operations of $\mathcal{K}$ on $\alpha(k, l)$, we find the following set of algebraically indecomposable primitives in $\mathcal{P}$ :

$$
\begin{aligned}
& \left\{d_{1}^{r} d_{p+1}^{s} \alpha(k, l) \mid r, s \geq 0 \text { and } 1 \leq k, l \leq p-1\right\}= \\
& \left\{( - 1 ) ^ { r } \left(k a_{k p^{s+\left(p^{s}-1\right)}}^{p^{r+1}} a_{l p^{r+s+1}+\left(p^{r+s+2}-1\right)}\right.\right. \\
& \left.\left.\quad-l a_{k p^{s}+\left(p^{s+1}-1\right)}^{p^{r+1}} a_{l p^{r+s+1}+\left(p^{r+s+1}-1\right)}\right) \mid r, s \geq 0 \text { and } 1 \leq k, l \leq p-1\right\},
\end{aligned}
$$

of length $p^{r+1}+1$.

Remark For $p=2$, the primitives in Example 4.3 were first obtained by Singer [21].

Example 4.4 (More relationships between $\alpha$ 's and $\beta$ 's in $\mathcal{P}$ ) Finally we are ready to consider the action of $d_{1}$ on all the primitives $d_{2}^{n} \beta(k, l)$. For $n=0$ this yielded $-\alpha(k, l)+\alpha(l, k)$ from Example 4.3. We intend to generalize this phenomenon. To calculate $d_{1} d_{2}^{n}(\beta(k, l))$ for $n \geq 1$ efficaciously, we first note that in $\mathcal{K}, d_{p+1} d_{1}=d_{1} d_{2}$ is easily computed from the $\mathcal{K}$ Adem relations given earlier. Using this on $d_{1} d_{2}^{n} \beta(k, l)$, we can repeatedly pass $d_{1}$ across $d_{2}$, replacing $d_{2}$ each time with $d_{p+1}$, producing

$$
d_{1} d_{2}^{n} \beta(k, l)=d_{p+1}^{n} d_{1} \beta(k, l)=-d_{p+1}^{n} \alpha(k, l)+d_{p+1}^{n} \alpha(l, k) .
$$

Thus we obtain for the $d_{1} d_{2}^{n} \beta(k, l)$ a linear combination of primitives derived at the end of Example 4.3 with $r=0$. 


\section{Proof of the main theorem}

We begin with motivation for the action defined above in the theorem.

Recall that the infinite loop space multiplication on $B U$ (or $B O$ ) makes $M$ into a Hopf algebra over the Dyer-Lashof algebra $\mathcal{R}$, or equivalently over the Kudo-Araki-May bialgebra $\mathcal{K}$. To get new primitives in $M$ from known ones, we might naïvely hope to use the Nishida relations (see Kochman [12]) connecting the standard $\mathcal{R}$ and $\mathcal{A}$ actions:

$$
\left(Q^{r}(x)\right) * P^{s}=\sum_{i}(-1)^{i+s}\left(\begin{array}{c}
(p-1)(r-s) \\
s-p i
\end{array}\right) Q^{r-s+i}\left(x * P^{i}\right),
$$

noting that they imply that an element $x$ in the kernel of all $* P^{i}$ also has each $Q^{r}(x)$ in the kernel of all $* P^{s}$. But, of course, $* P^{0}$ is the identity, so there are no such $x$. We need to look more carefully at the structure of $M$ itself over $\mathcal{R}$ (equivalently over $\mathcal{K})$.

We begin by translating the Dyer-Lashof operations in the Nishida formula into operations in $\mathcal{K}$. We obtain

$$
\left(d_{j}\left(x_{n}\right)\right) * P^{s}=\sum_{i}\left(\begin{array}{c}
(p-1)(j+n-s) \\
s-p i
\end{array}\right) d_{j+p i-s}\left(x_{n} * P^{i}\right) .
$$

Our goal is to modify this $\mathcal{K}$-action so that it will produce new primitives from old. Recall from above that the $i=0$ term in the Nishida formula obstructs our goal, but observe that eliminating all but the the last term on the right, for which $i=s / p$, would yield

$$
\left(d_{j}\left(x_{n}\right)\right) * P^{s}=d_{j}\left(x_{n} * P^{s / p}\right),
$$

which would admirably achieve our goal. Thus we aim greatly to simplify the $\mathcal{K}$-action formula to this end.

According to Kochman [12], the values of the Dyer-Lashof operations on the polynomial generators of $M=H_{*}\left(B U ; \mathbb{F}_{p}\right)$ (or $H_{*}\left(B O ; \mathbb{F}_{2}\right)$ if $p=2$ ) are given, for $n \geq 1$, by

$$
Q^{r}\left(a_{n}\right)=(-1)^{r+n+1}\left(\begin{array}{c}
r-1 \\
n
\end{array}\right) a_{n+(p-1) r}+\text { decomposables. }
$$

In terms of $\mathcal{K}$, this is

$$
d_{j}\left(a_{n}\right)=-\left(\begin{array}{c}
n+j-1 \\
n
\end{array}\right) a_{p n+(p-1) j}+\text { decomposables. }
$$

We note in particular that $d_{1}\left(a_{n}\right)=-a_{p n+(p-1)}+$ decomposables, and since $d_{0}$ is always the $p$ th power we have $d_{0}\left(a_{n}\right)=a_{n}^{p}$. Calculation of the $\mathcal{A}$-action shows that the leading terms here are compatible with our aim above, so we make the vastly simplified definition of a new action in the main theorem above based just on these 
leading terms. In our definition of the new action, it also seems necessary for the higher $d_{j}$ to be zero on the $a_{n}$ in order to have length degree respected via $d_{i}: M_{l, n} \rightarrow$ $M_{l+(p-1)(l-i), p n+(p-1) i}$, as needed to prove Proposition 3.1. Henceforth we use only this action of the $\mathcal{K}$-generators on $M$. Our goal is to prove that this new definition respects the Adem relations, creating an actual action by $\mathcal{K}$ that preserves the subalgebra $\mathcal{P}$ of primitives.

Remark The alternative action could equivalently (but less conveniently) be defined using the Dyer-Lashof algebra by the formula

$$
Q^{j}\left(a_{n}\right)= \begin{cases}a_{n}^{p} & \text { if } j=n, \\ a_{p n+(p-1)} & \text { if } j=n+1, \text { and } \\ 0 & \text { otherwise. }\end{cases}
$$

The proof of our main theorem will be accomplished in two steps. The first is the verification that we have an actual action by $\mathcal{K}$. The second is the simplified Nishida formula we need to ensure that the primitives $\mathcal{P}$ form a sub $\mathcal{K}$-algebra under this action.

Proof of Theorem We shall use formal power series over $\mathbb{F}_{p}$. Let $d(u)=\sum_{i \geq 0} d_{i} u^{i}$, and note for applying the Cartan formula that it is grouplike, that is, $\Delta d(u)=d(u) \otimes$ $d(u)$. Let $a(y)=\sum_{j \geq 1} a_{j} y^{j}$, recalling that there is no $a_{0}$. Also let $a^{(p-1)}(y)$ denote the ( $p-1)$ st derivative of $a(y)$, and recall Wilson's Theorem to observe that

$$
a^{(p-1)}(y)=-\sum_{k \geq 0} a_{k p+(p-1)} y^{k p} .
$$

This looks much like $d_{1}\left(a\left(y^{p}\right)\right)$, but we should remove its first term $-a_{p-1}$ because there is no $a_{0}$ in $a\left(y^{p}\right)$, so we define $\bar{a}^{(p-1)}(y)=a^{(p-1)}(y)+a_{p-1}$. Then we can express the alternative $\mathcal{K}$-action on the generators of $M$ in terms of formal power series by

$$
d(u) a\left(y^{p}\right)=[a(y)]^{p}+u \bar{a}^{(p-1)}(y) .
$$

Also recall from [15] and [16] that the Adem relations in $\mathcal{K}$ can be expressed by the formal power series identity

$$
d(u) d\left((u-v)^{p-1} v\right)=d(v) d\left((u-v)^{p-1} u\right) .
$$

The proof will be in two steps.

Step 1 The $\mathcal{K}$-action formulas in the theorem satisfy the Adem relations in $\mathcal{K}$ and hence define a homology $\mathcal{K}$-algebra structure on $M$. 
Proof of Step 1 We first compute

$$
\begin{aligned}
d(u) d\left((u-v)^{p-1} v\right) a\left(y^{p^{2}}\right)= & d(u)\left\{\left[a\left(y^{p}\right)\right]^{p}+(u-v)^{p-1} v \bar{a}^{(p-1)}\left(y^{p}\right)\right\} \\
= & {\left[d(u) a\left(y^{p}\right)\right]^{p}+(u-v)^{p-1} v d(u) \bar{a}^{(p-1)}\left(y^{p}\right) } \\
= & {\left[(a(y))^{p}+u \bar{a}^{(p-1)}(y)\right]^{p}+(u-v)^{p-1} v\left(\bar{a}^{(p-1)}(y)\right)^{p} } \\
& +(u-v)^{p-1} v u d_{1} \bar{a}^{(p-1)}\left(y^{p}\right) \\
= & (a(y))^{p^{2}}+\left(u^{p}+(u-v)^{p-1} v\right)\left(\bar{a}^{(p-1)}(y)\right)^{p} \\
& +(u-v)^{p-1} v u d_{1} \bar{a}^{(p-1)}\left(y^{p}\right) .
\end{aligned}
$$

Now

$$
\begin{aligned}
u^{p}+(u-v)^{p-1} v & =\left(u^{p}-v^{p}\right)+(u-v)^{p-1} v+v^{p} \\
& =(u-v)^{p}+(u-v)^{p-1} v+v^{p} \\
& =(u-v)^{p-1} u+v^{p},
\end{aligned}
$$

that is, it is symmetric in $u$ and $v$. So $d(u) d\left((u-v)^{p-1} v\right) a\left(y^{p^{2}}\right)$ is symmetric in $u$ and $v$, and hence is equal to $d(v) d\left((u-v)^{p-1} u\right) a\left(y^{p^{2}}\right)$. Thus the Adem relations are satisfied on the polynomial generators, and so, by the definition of the $\mathcal{K}$-action on all of $M$ through the Cartan formula, they are satisfied on all of $M$.

Step 2 The $\mathcal{K}$-action formulas in the theorem satisfy the identity

$$
\left(d_{i} x\right) * P^{k}=d_{i}\left(x * P^{k / p}\right)
$$

for any element $x \in M$, and hence the primitives become a sub-K -algebra under the $\mathcal{K}$-action.

Proof of Step 2 We could prove this formula by brute force calculation with binomial coefficients. However, we prefer to continue with a formal power series approach. Let $P(z)=\sum_{r \geq 0} P^{r} z^{r}$, for which $\Delta P(z)=P(z) \otimes P(z)$. Note that the downward $\mathcal{A}$-action on $M$

$$
a_{m} * P^{r}=\left(\begin{array}{c}
m-r(p-1) \\
r
\end{array}\right) a_{m-r(p-1)}
$$

is expressed in formal power series by the identity

$$
a(x) * P\left(x^{-p} y\right)=a(x+y) .
$$

We remark that taking the $k$ th partial derivative with respect to $x$ of both sides of this equation yields

$$
\begin{aligned}
a^{(k)}(x) * P\left(x^{-p} y\right) & =a^{(k)}(x+y) \\
\text { whence } \quad \bar{a}^{(k)}(x) * P\left(x^{-p} y\right) & =\bar{a}^{(k)}(x+y) .
\end{aligned}
$$


We may now check the interaction of the $\mathcal{A}$-action with the $\mathcal{K}$-action essentially by verifying an identity of formal power series:

First we check the identity

$$
\left(d_{i} a_{j}\right) * P^{k}=d_{i}\left(a_{j} * P^{k / p}\right)
$$

via the formal power series computation

$$
\begin{aligned}
{\left[d(u) a\left(x^{p}\right)\right] * P\left(x^{-p} y\right) } & =\left[[a(x)]^{p}+u \bar{a}^{(p-1)}(x)\right] * P\left(x^{-p} y\right) \\
& =[a(x+y)]^{p}+u \bar{a}^{(p-1)}(x) * P\left(x^{-p} y\right) \\
& =[a(x+y)]^{p}+u \bar{a}^{(p-1)}(x+y) \\
& =d(u) a\left(x^{p}+y^{p}\right) \\
& =d(u)\left[a\left(x^{p}\right) * P\left(x^{-p^{2}} y^{p}\right)\right] .
\end{aligned}
$$

Now the identity for general $x \in M$ follows by calculation from the identity above for the $a_{j}$, since both the $\mathcal{A}$-action and the $\mathcal{K}$-action satisfy Cartan formulas.

This completes the proof of the theorem.

\section{Closing comments}

Remark (Singer's action of the bigraded Steenrod algebra) A graded cohomology $\mathcal{K}$-module $M$ is one in which the action satisfies $\mathcal{K}_{m, r} \otimes M_{S} \rightarrow M_{p^{m_{s}-r}}$. Such an action by $\mathcal{K}$ determines an action by Steenrod operations $[15 ; 16]$. When $p=2$, this takes the form $\mathrm{Sq}^{i} x=d_{r-i} x$, where $x$ is a class of grade $r$, for an appropriate grading. If we take for our grading the length grading in $M$, then the formulas for our $\mathcal{K}$-action do clearly create a graded cohomology $\mathcal{K}$-module structure on $M$, and translate to

$$
\mathrm{Sq}^{i}\left(a_{n}\right)= \begin{cases}a_{n}^{2} & \text { if } i=1, \\ a_{2 n+1} & \text { if } i=0, \text { and } \\ 0 & \text { if } i>1,\end{cases}
$$

since $\operatorname{Sq}^{i}\left(a_{n}\right)=d_{1-i}\left(a_{n}\right)$ for all generators. This is the action created by Singer [21] of his "bigraded Steenrod algebra" (note that $\mathrm{Sq}^{0}$ acts nontrivially, so this is not an action of the Steenrod algebra $\mathcal{A}$ ).

We have here a highly curious and unusual situation with the bigraded $M$. The single $\mathcal{K}$-module structure we have defined makes $M$ simultaneously into a graded cohomology $\mathcal{K}$-module with respect to its length grading, and a graded homology 
$\mathcal{K}$-module with respect to its topological grading, as noted earlier. Taken together this is what enables $M$ to have essentially equivalent actions of both the Dyer-Lashof algebra and Singer's bigraded Steenrod algebra, so that the two actions produce the same primitives.

Remark (The Peterson conjecture and odd primes) A natural odd primary "Peterson conjecture" for $M$ is that $\mathcal{P}$, in length degree $n$, is concentrated in topological degrees $d$ such that $d+n$ has no more than $n$ non-zero digits in its $p$-ary expansion, since this has been verified for $p=2$ by Janfada and Wood [9]. Moreover, our results here are consonant at all primes with this conjecture, in that action by any $d_{i}$ multiplies the total degree $d+n$ by $p$. Although this conjecture is false in general for odd primes, in a future paper [14] we shall show that it holds for $H^{*}\left(B U(2) ; \mathbb{F}_{3}\right)$, and in somewhat altered form for $H^{*}\left(B U(2) ; \mathbb{F}_{p}\right), p \geq 5$.

\section{References}

[1] M A Alghamdi, M C Crabb, J R Hubbuck, Representations of the homology of $B V$ and the Steenrod algebra I, from: "Adams Memorial Symposium on Algebraic Topology, 2 (Manchester, 1990)", London Math. Soc. Lecture Note Ser. 176, Cambridge Univ. Press, Cambridge (1992) 217-234 MR1232208

[2] J M Boardman, Modular representations on the homology of powers of real projective space, from: "Algebraic topology (Oaxtepec, 1991)", Contemp. Math. 146, Amer. Math. Soc., Providence, RI (1993) 49-70 MR1224907

[3] R R Bruner, L M Hà, N H V Hung, On the behavior of the algebraic transfer, Trans. Amer. Math. Soc. 357 (2005) 473-487 MR2095619

[4] D P Carlisle, R M W Wood, The boundedness conjecture for the action of the Steenrod algebra on polynomials, from: "Adams Memorial Symposium on Algebraic Topology, 2 (Manchester, 1990)", London Math. Soc. Lecture Note Ser. 176, Cambridge Univ. Press, Cambridge (1992) 203-216 MR1232207

[5] M C Crabb, J R Hubbuck, Representations of the homology of BV and the Steenrod algebra II, from: "Algebraic topology: new trends in localization and periodicity (Sant Feliu de Guíxols, 1994)”, Progr. Math. 136, Birkhäuser, Basel (1996) 143-154 MR1397726

[6] MD Crossley, $\mathcal{A}(p)$-annihilated elements in $H_{*}\left(\mathbb{C} \mathrm{P}^{\infty} \times \mathbb{C} \mathrm{P}^{\infty}\right)$, Math. Proc. Cambridge Philos. Soc. 120 (1996) 441-453 MR1388199

[7] L M Hà, Sub-Hopf algebras of the Steenrod algebra and the Singer transfer, from: "Proceedings of the School and Conference in Algebraic Topology", Geom. Topol. Monogr. 11, Geom. Topol. Publ., Coventry (2007) 81-105 MR2402802 
[8] N H V Hung, The cohomology of the Steenrod algebra and representations of the general linear groups, Trans. Amer. Math. Soc. 357 (2005) 4065-4089 MR2159700

[9] A S Janfada, R M W Wood, The hit problem for symmetric polynomials over the Steenrod algebra, Math. Proc. Cambridge Philos. Soc. 133 (2002) 295-303 MR1912402

[10] A S Janfada, R M W Wood, Generating $H^{*}\left(\mathrm{BO}(3), \mathbb{F}_{2}\right)$ as a module over the Steenrod algebra, Math. Proc. Cambridge Philos. Soc. 134 (2003) 239-258 MR1972137

[11] M Kameko, Generators of the cohomology of $B V_{3}$, J. Math. Kyoto Univ. 38 (1998) 587-593 MR1661173

[12] S O Kochman, Homology of the classical groups over the Dyer-Lashof algebra, Trans. Amer. Math. Soc. 185 (1973) 83-136 MR0331386

[13] T N Nam, $\mathcal{A}$-générateurs génériques pour l'algèbre polynomiale, Adv. Math. 186 (2004) 334-362 MR2073910

[14] D J Pengelley, F Williams, The hit problem for $H^{*}\left(B U(2) ; \mathbb{F}_{p}\right)$, in progress

[15] D J Pengelley, F Williams, Sheared algebra maps and operation bialgebras for mod 2 homology and cohomology, Trans. Amer. Math. Soc. 352 (2000) 1453-1492 MR1653375

[16] D J Pengelley, F Williams, The odd-primary Kudo-Araki-May algebra of algebraic Steenrod operations and invariant theory, from: "Proceedings of the School and Conference in Algebraic Topology”, Geom. Topol. Monogr. 11, Geom. Topol. Publ., Coventry (2007) 217-243 MR2402808

[17] F P Peterson, Generators of $H^{*}\left(\mathbb{R} \mathrm{P}^{\infty} \wedge \mathbb{R P}^{\infty}\right)$ as a module over the Steenrod algebra, Abstracts Amer. Math. Soc. 833-55-89 (April 1987)

[18] F P Peterson, A-generators for certain polynomial algebras, Math. Proc. Cambridge Philos. Soc. 105 (1989) 311-312 MR974987

[19] G M L Powell, Embedding the flag representation in divided powers, J. Homotopy Relat. Struct. 4 (2009) 317-330 MR2559645

[20] W M Singer, The transfer in homological algebra, Math. Z. 202 (1989) 493-523 MR1022818

[21] W M Singer, Rings of symmetric functions as modules over the Steenrod algebra, Algebr. Geom. Topol. 8 (2008) 541-562 MR2443237

[22] R M W Wood, Steenrod squares of polynomials and the Peterson conjecture, Math. Proc. Cambridge Philos. Soc. 105 (1989) 307-309 MR974986

New Mexico State University, Las Cruces NM 88003, USA

davidp@nmsu.edu, frank@nmsu.edu

http://www.math.nmsu.edu/ davidp

Received: 10 July 2010 Revised: 9 February 2011 